This document is the accepted manuscript version of the following article:

Xiao, F., Zheng, P., Tria, J. D., Kocer, B. B., \& Kovac, M. (2021). Optic flow-based

reactive collision prevention for MAVs using the fictitious obstacle hypothesis. IEEE

Robotics and Automation Letters, 6(2), 3144-3151. https://doi.org/10.1109/LRA.2021.3062317

\title{
Optic Flow Based Reactive Collision Prevention for MAVs Using the Fictitious Obstacle Hypothesis
}

\author{
Feng Xiao ${ }^{1}$, Peter Zheng ${ }^{1,2}$, Julien di Tria ${ }^{1}$, Basaran Bahadir Kocer ${ }^{1}$ and Mirko Kovac ${ }^{1,3}$
}

\begin{abstract}
Optical flow sensors and optical flow divergence (OFD) have offered partial solutions for obstacle avoidance, landing, and perching with micro aerial vehicles. Theoretically, OFD can indicate the risk of collision, providing that the sensors' field of view is bounded within a single flat surface on the obstacle. However, in the real world, directly measuring the risk of collision with OFD generates false alarms due to rapidly changing speeds and irregular surroundings. In this letter, we present a new obstacle detection strategy based on an extended Kalman filter (EKF) combining the OFD with inertial sensing. The introduction of a fictitious obstacle hypothesis and the use of the EKF estimates enable us to differentiate the surrounding-generated OFD from the OFD caused by the actual obstacle. An embedded constant zero-OFD controller is then used for post-detection emergency deceleration. The ultra-light OFD estimation and control system, with a mass of $20 \mathrm{~g}$, estimates OFD at $160 \mathrm{~Hz}$. The system was validated on a $158 \mathrm{~g}$ mini quadrotor in both laboratory and field tests. Experimental results illustrate that the presented system can achieve accurate obstacle detection, near-obstacle distance estimation, and controlled deceleration to prevent collisions [Video attachment: https://youtu.be/yIyYHYNOjOw].
\end{abstract}

Index Terms-Aerial Systems: Applications; BiologicallyInspired Robots; Collision Avoidance.

\section{INTRODUCTION}

$\mathbf{O}$ BSTACLE detection and collision prevention during autonomous missions has been an ongoing area of research for micro aerial vehicles (MAVs) with limited payload, computation, and communication capabilities. While state estimation and path planning mitigate the risks, there is a need for an embedded low-level system for sudden reactions. We envision a primary collision prevention system that interrupts

Manuscript received: October 15, 2020; Revised December 31, 2020; Accepted February 8, 2021.

This paper was recommended for publication by Editor-in-Chief Allison Okamura and Editor Pauline Pounds upon evaluation of the Associate Editor and Reviewers' comments. This work was supported in part by EPSRC (award no. EP/R009953/1, EP/N018494/1, and EP/R026173/1), NERC (grant number NE/L002515/1), SEARRP, and the EU H2020 AeroTwin project (grant ID 810321). M. Kovac is supported by the Royal Society Wolfson fellowship (RSWF/R1/18003). The Multi-Terrain Aerial Robotics Arena is supported through a philanthropic gift by Brahmal Vasudevan.

${ }^{1}$ Feng Xiao, Peter Zheng, Julien di Tria, Basaran Bahadir Kocer and Mirko Kovac are with the Aerial Robotics Lab (ARL), Department of Aeronautics, Imperial College London, South Kensington Campus, London, SW7 2AZ United Kingdom feng.xiao16@imperial.ac.uk, peter.zheng130imperial.ac.uk,

j.di-tria20@imperial.ac.uk, b.kocer@imperial.ac.uk, m.kovac@imperial.ac.uk

${ }^{2}$ Peter Zheng is also with the Grantham Institute, Imperial College London, South Kensington Campus, London, SW7 2AZ United Kingdom

${ }^{3}$ Mirko Kovac is also with the Swiss Federal Laboratories for Materials Science and Technology (EMPA), Ueberlandstrasse 129, 8600 Dübendorf, Switzerland http://robotics.empa.ch

Digital Object Identifier (DOI): see top of this page. the high-level controller and performs control actions fully onboard when danger is detected. Our concept is developed to ensure the survivability of the platform, upon which secondary systems such as vision-based navigation can be added to ensure the continuation of the mission.

Commonly used state estimation and obstacle detection equipment, such as RADAR/LIDAR [1], [2], cameras [3], [4], and event-based cameras [5], require a combination of significant payload capacity and onboard computation. Time of flight $(\mathrm{ToF})$ sensors provide single direction distance sensing. Thus, a single sensor cannot detect clusters of sparse obstacles such as fences and bushes. The weight of these ToF sensors increases with their accuracy and sampling rate. Lightweight options such as the VL53L0x weigh less than $1 \mathrm{~g}$ but may have low sampling rates $(10 \mathrm{~Hz})$. Therefore, lightweight ToF sensors are neither reliable nor fast enough for real world applications.

Small flying insects neither measure the absolute distances to objects nor their own speed [6]. Instead, they use optical flow $(\mathrm{OF})$, which is the 2-dimensional visual movement patterns on their retina due to the 3-dimensional (3D) motion of flight. This intrinsic information is sufficient to obtain the visual cues containing the relative velocity without a length scale [7], [8]. McGuire et al. designed a $40 \mathrm{~g}$ drone with a specialized stereo camera that could estimate distance and optical flow, and derive its own velocity, with which obstacle avoidance was demonstrated in a room at low speed [9]. Due to the sensor's low sampling rate of $30 \mathrm{~Hz}$, the maximum safe airspeed is limited.

The reciprocal of the time-to-contact can be obtained from the optical flow divergence (OFD) and be used for obstacle avoidance [10]-[12]. One can set two OFD thresholds to detect front and side obstacles in complex 3D environments [13]. OFD has also been used as the observation update in an extended Kalman filter (EKF) to estimate the distance and velocity of a MAV during landing [14]. The constant OFD strategy has been used for smooth landing control [12], [15], [16]. Overall, using OFD for obstacle detection is computationally efficient and lightweight, making it suitable for the primary collision prevention role described above.

While OFD can be calculated with a single camera using computer vision libraries such as OpenCV, it can also be calculated with two cameras facing $\pm 45^{\circ}$ about the vehicle's yaw axis [17]-[19]. This method has been implemented on both ground vehicles [11] and fixed-wing aerial vehicles [20]. When using OF sensors which can directly output integrated OF data across the image plane, image processing and onboard computers are not required. In this letter, the pair of OF sensors are named divergent optical flow pair (DOFP). In this 

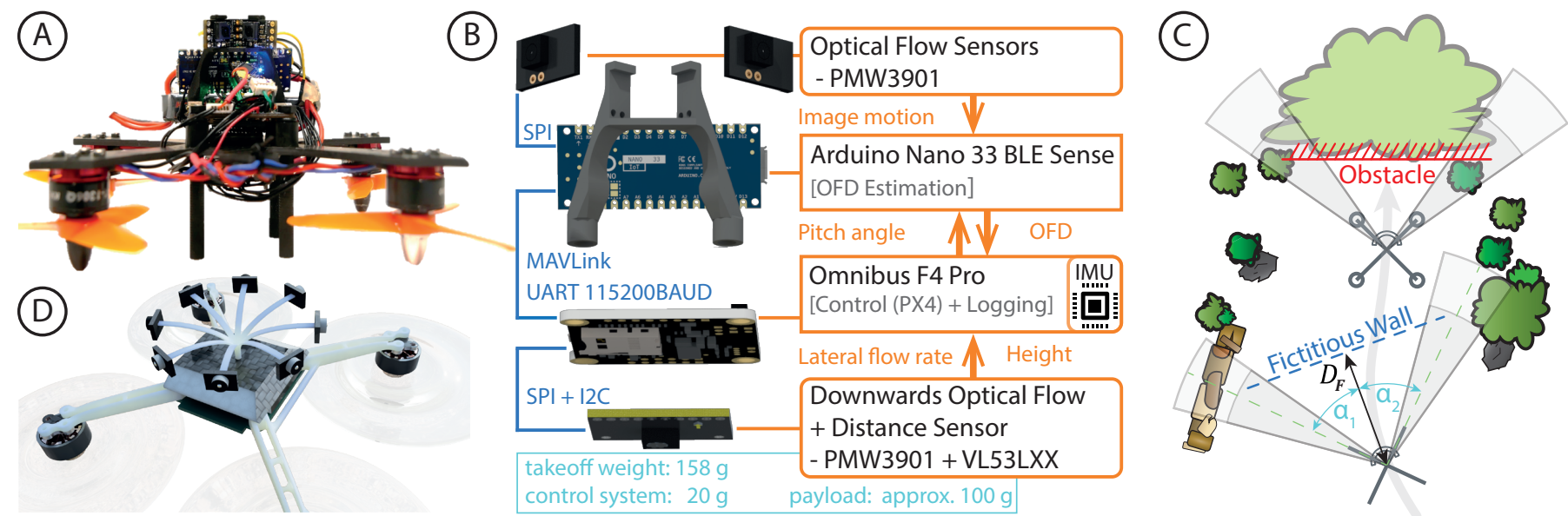

Fig. 1. Overview of the design. (A) Picture of the prototype MAV. (B) The electronics system. The optical flow sensors and the microcontroller are mounted on a 3D-printed supporting structure. (C) The fictitious wall concept. (D) Concept drawing of a $360^{\circ}$ ring of sensors for obstacle detection in all directions.

context, the testing environments in previous implementations are highly idealized. For the use of DOFP and OFD for primary collision prevention to be fully realized, we must systematically characterize the performance and limitations of DOFP in the real world and gain further insights into how to best optimize its use.

The use of DOFP for obstacle detection presents the following challenges: a) The estimation algorithm is based on a flat surface that is large enough to cover the sensors' field of view $(\mathrm{FoV})$. This limits the usage of the OFD. b) Using an OFD constant as the obstacle detection criterion is simple but this only works when the flight velocity is relatively constant. For aggressive maneuvers, this criterion can easily generate a false positive alarm and interrupt the mission.

In this work, the real world implications of using DOFP are investigated for OFD estimation, obstacle detection, and collision prevention (Section IIII). We characterize the effectiveness of the OFD estimation method when the obstacle does not fully cover the DOFP's FoV and introduce the concept of a fictitious wall to dispense with the flat surface assumption (Section IV]. This is further developed into an obstacle detection strategy based on the EKF estimates (Section $\mathrm{V}$ ). The OFD estimation is then used in a constant zero-OFD controller [21] to control the emergency deceleration when triggered by the obstacle detection system. To validate our framework, we designed a MAV and DOFP prototype (Section [II). The results from laboratory and outdoor tests and discussions are presented (Section VI).

\section{MODELING AND PROTOTYPE DESIGN}

\section{A. Coordinate Systems}

While an aerial collision can occur from all directions, it is most likely to occur in the direction of travel. Hence, we define the geometry of the problem with a quadrotor that flies forward, and a static wall as the obstacle (Fig. 2). The body frame of the MAV is fixed at $O^{b}$, the MAV's center of mass. Following the right-hand rule, $X^{b}$ points in the forward direction of the MAV, and $Z^{b}$ downward. $X^{b}$ and $Y^{b}$ are fixed on the MAV's airframe. The frame $O^{b^{\prime}} X^{b^{\prime}} Y^{b^{\prime}} Z^{b^{\prime}}$ shares the
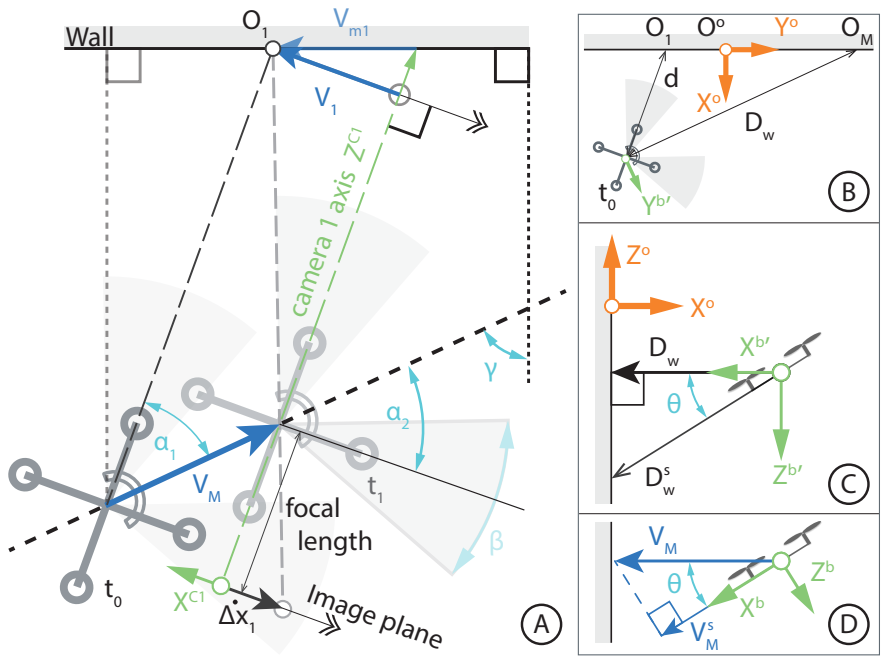

Fig. 2. The coordinate system and geometry relations used by the OFD estimation. (A,B) Top view of the MAV flying towards a flat vertical surface. All the labeled vectors are on the $X^{O} Y^{O}$ plane. (C,D) Side view of the MAV. For a clear illustration, only the velocities related to camera 1 are shown.

same center as $O^{b}$. The $X^{b^{\prime}}$ and $Y^{b^{\prime}}$ axes are $X^{b}$ and $Y^{b}$ projected on the horizontal plane; $Z^{b^{\prime}}$ axis points downward. The frame $O^{O} X^{O} Y^{O} Z^{O}$ is located on the surface of the flat vertical wall. $\left(X^{O}-Y^{O}\right)$ is parallel to the horizontal surface. Each of the two OF camera lenses has a FoV of $\beta$. Their axes, $Z^{C 1}$ and $Z^{C 2}$, have offsets $\alpha_{1}$ and $\alpha_{2}$ from $X^{b}$ respectively. $V_{M}$ is the MAV velocity along $X^{b^{\prime}}$ axis and is positive when the MAV flies forward.

The MAV may tilt in numerous directions. Thus, the flight direction may not coincide with $X^{b^{\prime}}$. However, the flight direction can be obtained by the velocities along $X^{b^{\prime}}$ and $Y^{b^{\prime}}$ axis. With a conceptual extended design (Fig. 1D), we can activate the two sensors closest to the flight direction. In our approach, direction $X^{b^{\prime}}$ is considered as the flight direction for simplicity. Therefore, the roll angle is negligible. 


\section{B. Electronic System}

The DOFP features two PMW3901 OF sensors, mounted on top of the electronics stack to ensure a clear line of sight (Fig. 1), and near $O^{b}$ to match our theoretical model (Fig. 2). These sensors can output the integrated pixel movements across the image plane. The microcontroller computes the OFD and the autopilot runs a modified PX4 firmware with the obstacle detection framework and constant zero-OFD controller. The downward-facing optical flow and distance sensor provide the data to maintain altitude and minimize horizontal drift.

\section{OFD ESTIMATION AND CONSTANT ZERO-OFD CONTROL}

\section{A. Estimation on an Infinitely Long Wall}

OFD is defined as:

$$
\mathrm{OFD}=\frac{V_{M}}{D_{w}}
$$

where $D_{w}$ is the distance to the wall from $O^{b}$ in the $X^{b^{\prime}}$ axis (Fig. 2). $\gamma$ is the incidence angle between the MAV flight path and the axis normal to the wall, $X^{O}$.

The OF sensor outputs pixel motion counts $\Delta x_{1}$ and $\Delta y_{1}$ in $X^{c 1}$ and $Y^{c 1}$ axes of the image plane. They represent the relative movement detected by the sensor during one time step. From $t_{0}$ to $t_{1}$, point $O_{1}$ on the wall seen by the left OF sensor moves by $\Delta x_{1}$ on the image plane. The related velocity vector is represented as $\Delta x_{1}$, which is projected on the image plane by $V_{m 1}$ on the wall or $V_{1}$ on the virtual plane parallel to the image plane crossing $O_{1}$.

$$
V_{1}=V_{m 1} \cos \left(\gamma-\alpha_{1}\right)
$$

As the time step is small, we treat these velocities as velocities at $t_{0}$.

The angular velocity of the pixel motion with respect to the focal length is called optical flow rate $\Lambda_{x 1} . \Lambda_{x 1}=\frac{\Delta x_{1}}{f}$, where $f$ is a scaling factor related to the pixel size and the focal length of the OF sensor that transforms the unit "pixel/second" to "radian/second". The OF sensor is treated as a black box. $f$ was determined by comparing OF sensor data with motion capture (mocap) ground truth.

The angular velocity related to $V_{1}$ with respect to the $\mathrm{OF}$ sensor origin is $\omega_{1}$, which equals to its measured flow rate $\Lambda_{x 1}$ :

$$
\omega_{1}=\frac{V_{1}}{d}=\Lambda_{x 1}
$$

Based on the geometric relations and the sine rule:

$$
\begin{aligned}
d & =\frac{D_{w} \cos \gamma}{\cos \left(\gamma-\alpha_{1}\right)} \\
\frac{V_{m 1}}{V_{M}} & =\frac{\left|O_{1} O_{M}\right|}{D_{w}}=\frac{\sin \alpha_{1}}{\sin \left(\pi / 2+\gamma-\alpha_{1}\right)}
\end{aligned}
$$

From above equations:

$$
\Lambda_{x 1}=\text { OFD } \frac{\sin \alpha_{1}}{\cos \gamma} \cos \left(\gamma-\alpha_{1}\right)
$$
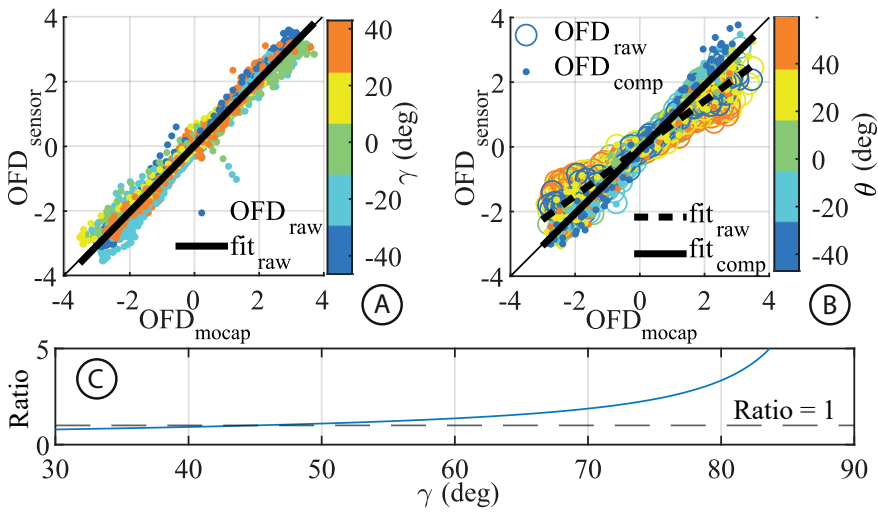

Fig. 3. Cross correlation plots between the OFD ground truth ("mocap" subscript) and OFD estimation ("sensor") with (A) incidence angle $\gamma$, and (B) before and after pitch compensation ("comp"), showing the data for different $\gamma$ and $\theta$ (color bar to represent different values), and the linear regression curve ("fit"); (C) the ratio of OFD estimated by a single OF sensor to the theoretical OFD when MAV approaching the wall with large incidence angle $\gamma$. The ratio increases exponentially when $\gamma \rightarrow 90^{\circ}$.

where $\Lambda_{x 1}$ is estimated by the OF sensor, and $\alpha_{1}$ is specified in the platform design. With the two OF sensors mounted at $\alpha_{1}=\frac{\pi}{4}$ and $\alpha_{2}=-\frac{\pi}{4}$, OFD can be calculated by

$$
\mathrm{OFD}=\Lambda_{x 1}-\Lambda_{x 2}
$$

\section{B. Adjustment for Quadrotor Platform}

The underactuated MAV must pitch to fly forward (Fig.2B). This induces an error in the OFD estimation. The OFD estimated by the sensor, $\mathrm{OFD}^{\mathrm{s}}$, is corrected by the MAV pitch angle $\theta$ such that

$$
\mathrm{OFD}=\frac{V_{M}}{D_{w}}=\frac{V_{M}{ }^{\mathrm{s}} / \cos \theta}{D_{w}{ }^{\mathrm{s}} \cos \theta}=\frac{\mathrm{OFD}^{\mathrm{s}}}{\cos ^{2} \theta}
$$

We used a mocap system to obtain the ground truth of the OFD for comparison with the DOFP estimated OFD. A complementary low-pass filter was added to reduce the signal noise. (7) implies that the OFD estimation does not depend on the incidence angle $\gamma$. This was further verified experimentally (Fig. 3). The estimated OFD has a small root mean square error of $0.3274 \mathrm{~s}^{-1}$ compared with the ground truth data ranging from $-3.5 \mathrm{~s}^{-1}$ to $3.75 \mathrm{~s}^{-1}$.

\section{Single OF Sensor Estimation}

As incidence angle $\gamma$ increases, the wall gradually disappears in one of the OF sensors' FoV. When $\gamma>\alpha_{1(2)}+\beta / 2$, one OF sensor does not see the wall. In the real world, where the wall is not infinitely long, this critical angle will be lower. In such cases, the OFD estimation with DOFP becomes invalid. However, if the surrounding environment is clear, i.e. the OF sensor looking out of the wall gives near-zero flow rate, (7) will become $O \hat{F} D \approx \Lambda_{x 1}\left(\Lambda_{x 2} \approx 0\right)$. Thus, with (6), we have:

$$
\frac{O \hat{F} D}{O F D} \approx \frac{\sin \alpha_{1}}{\cos \gamma} \cos \left(\gamma-\alpha_{1}\right)
$$

which shows that the approximated OFD and real OFD have a ratio which varies with $\gamma$ (Fig. $3 \mathrm{C})$. Although the estimation 
is not accurate, a single OF sensor can generate a scaled estimation of the OFD, and the ratio is near 1 in the range of $30^{\circ}$ to $60^{\circ}$. However, an accurate OFD estimation depends on the $\gamma$, which is unknown to a single OF sensor system.

\section{Constant Zero OFD Control}

The constant OFD strategy has been widely used for docking and landing. By keeping the OFD constant, $\frac{V_{M}}{D_{w}}=\lambda$ :

$$
D_{w}=D_{w_{0}} e^{-\lambda t}, V_{M}=-\dot{D}_{w}=\lambda D_{w_{0}} e^{-\lambda t}
$$

Therefore, if $\lambda>0, D_{w}$ and $V_{M}$ will converge to zero. This is ideal for scenarios where the velocity needs to decrease gradually when approaching the surface. However, constant zero OFD would be more beneficial for obstacle avoidance. If $\lambda=0$, (10) reduces to $D_{w}=D_{w_{0}}$ and $V_{M}=0$, which means the MAV will hold a constant distance to the obstacle.

The forward position along the $X^{b^{\prime}}$ axis is controlled by a proportional controller to maintain $\mathrm{OFD}=0$. The altitude and lateral motion are controlled by proportional-integralderivative (PID) controllers to keep the MAV flying straight at a constant height.

\section{SYSTEM CHARACTERISTICS AND FICTITIOUS WALL HYPOTHESIS}

\section{A. Close-field and Far-field Estimation}

Infinitely long walls do not exist in the real world. However, this is not strictly required for the OFD estimation algorithm to generate useful data. The whole flow field changes when the MAV moves, thus providing sufficient features in the image plane. When objects within the FoV move and the MAV stays static, only a part of the flow field changes. Depending on the percentage of the FoV covered by the changing flow field, the reliability of the OFD estimation may be adversely affected.

An object of width $L$ at distance $D$ in front of the MAV ( $\gamma \approx 0^{\circ}$, and MAV centered) covers a percentage $c$ of the DOFP FoV where

$$
\begin{aligned}
c & = \begin{cases}0 & \text { if } \phi<\phi_{1} \\
\left(\phi-\phi_{1}\right) / \beta, & \text { if } \phi_{1} \leq \phi \leq \phi_{2} \\
1, & \text { if } \phi>\phi_{2}\end{cases} \\
\phi_{1,2} & =\alpha \mp \beta / 2 \\
\phi & =\arctan (L /(2 D))
\end{aligned}
$$

The effects of a moving MAV with a static wall and a static MAV with a moving wall were studied against a $2 \mathrm{~m}$ wide, $1.2 \mathrm{~m}$ high vertical wall. The MAV followed a $\pm 0.25 \mathrm{~m}$ square wave position set-point along the $X^{b^{\prime}}$ axis with ten different starting points, ranging from 0.5 to $5 \mathrm{~m}$. The Pearson correlation coefficients at different distances indicate that within $1.5 \mathrm{~m}$ of the wall, the OFD estimation is always valid (Fig. (4).

As $\left|\alpha_{1}-\alpha_{2}\right|=90^{\circ}, \beta=42^{\circ}$, the wall covers $23 \%$ of the DOFP FoV at $1.5 \mathrm{~m}$ according to (11). The effectiveness of the moving wall situation drops sharply if the relevant distance is further than $2 \mathrm{~m}$, when $c \leq 5 \%$.

Beyond $4.5 \mathrm{~m}(c=0)$, the moving wall and moving MAV scenarios exhibit different behaviors (Fig. $4 \mathrm{~B} / \mathrm{C}$ ). When the
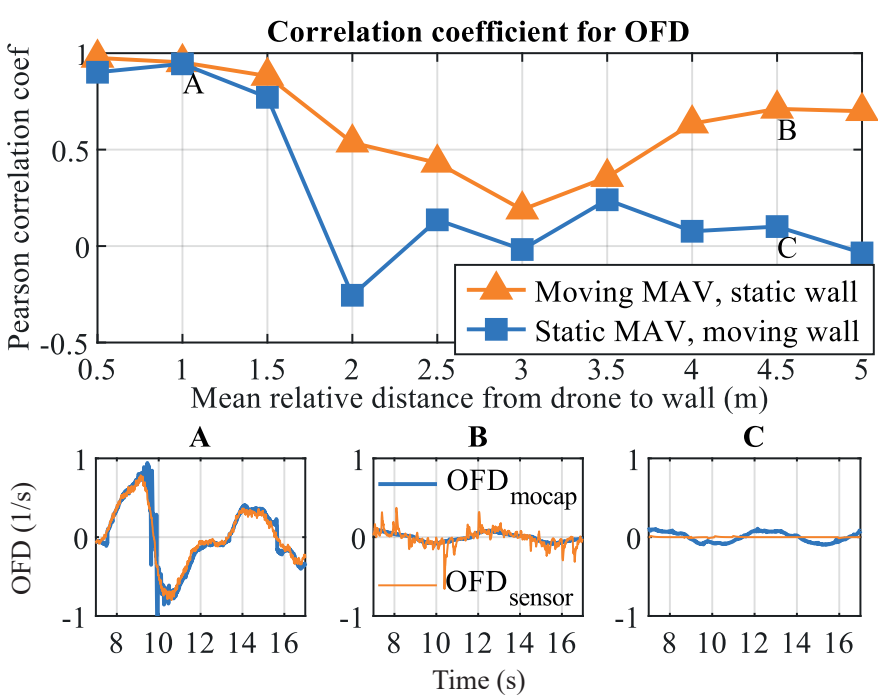

Fig. 4. Top: the correlation coefficient between the OFD estimation and the ground truth at different relative distances. The correlation coefficient is a unit-less value. The closer to 1 , the more effective the estimation is. Bottom: mocap OFD and sensor OFD data comparison on three selected cases labelled as $\mathrm{A}, \mathrm{B}$, and $\mathrm{C}$ in the top figure.

wall moves, the sensor OFD stays constant at $0 \mathrm{~s}^{-1}$ as no motion is captured. When the MAV moves, although the DOFP does not see the wall, surrounding features are observed. This generates noisy but representative data that follows the MAV's oscillations as if there was a wall in front. Thus, the correlation is higher in the moving MAV scenario. This behavior shows that far-field surroundings can be approximated by a flat wall, relaxing the restriction of the physical flat wall obstacle assumption.

\section{B. Fictitious Wall}

The far-field surroundings can resemble a flat wall. However, the "wall" and its location are immaterial. Thus, we introduce the concept of the fictitious wall. The fictitious wall (Fig. 1C) is defined as a virtual wall located in front of the quadrotor with a $D_{F}$ distance at time $t$.

$$
\left.D_{F}\right|_{t}=\frac{\left.V_{M}\right|_{t}}{\left.O F D\right|_{t}}
$$

At each $t$, the OFD estimation will be equal to observing a static wall located at $\left.D_{F}\right|_{t}$ in front of the MAV. The fictitious wall is static at each $t$, and the velocity of the fictitious wall is $0 \mathrm{~m} \mathrm{~s}^{-1}$ (Section $\mathrm{V}$-E).

\section{OFD Threshold as Obstacle Detection Criterion}

When the flight speed is relatively constant, an OFD threshold can be used as the obstacle detection criterion. However, during dynamic flights with aggressive maneuvers, a simple constant threshold may generate false positives. Furthermore, there are cases where DOFP-estimated OFD does not indicate the risk of collision. For example, when MAV flies along a clear path with objects on both sides, neither a small nor a large value of OFD necessarily indicates an obstacle ahead.

We propose the fictitious obstacle hypothesis to differentiate between real frontal obstacles and fictitious obstacles: 1) the 


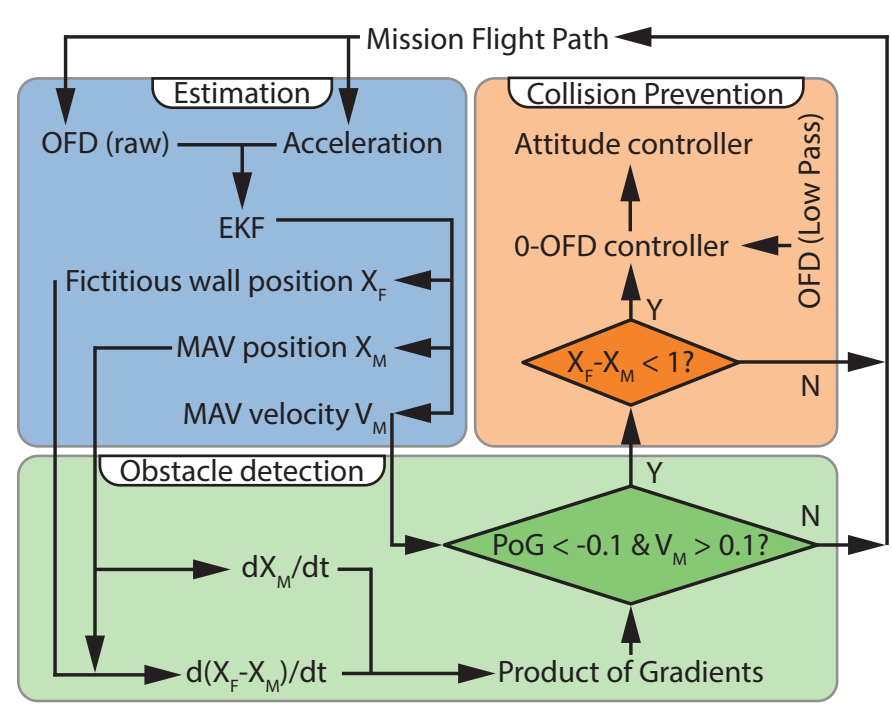

Fig. 5. A flow chart showing the EKF estimation, the obstacle detection and the collision prevention process.

fictitious wall should move forward along with the MAV until the obstacle covers most of the DOFP's FoV. Then the fictitious wall should coincide with the actual obstacle and stop moving. 2) OFD is a relative value affected by both the MAV's motion and the environment. Acceleration from the autopilot IMU represents MAV's motion and can be used to decouple the motion and the environment.

With the acceleration and OFD, we designed a 1D EKF to estimate the fictitious wall location, the distance between the fictitious wall and the MAV, and the MAV's velocity.

\section{Static obstacle Detection With Fictitious WALL THEORY}

\section{A. Extended Kalman Filter}

EKF has been used for multirotor landing control [14], with the control acceleration command being the model input and the OFD being the observation update. The state vector includes just the velocity and the height. As the OFD measurement is in the height direction and the floor is a large static surface, the flat surface assumption is valid.

However, for forward flight, as we are considering a fictitious wall that is always changing in location until the obstacle becomes observable, we constructed an EKF using acceleration from the IMU as model input and divided the distance into two variables: the location of the MAV $X_{M}$ and the location of the fictitious wall $X_{F}$. The 1D continuous model dynamics can be written as

$$
\begin{aligned}
& \dot{\boldsymbol{x}}=\left[\begin{array}{lll}
\dot{X}_{M} & \dot{V}_{M} & \dot{X}_{F}
\end{array}\right]^{T}=\left[\begin{array}{ccc}
V_{M} & a & 0
\end{array}\right]^{T} \\
& y=h(\boldsymbol{x})=O F D=\frac{V_{M}}{D_{F}}=\frac{V_{M}}{X_{F}-X_{M}}
\end{aligned}
$$

where $a$ is the acceleration along $X^{b^{\prime}}$, calculated by compensating the IMU value with the pitch angle. The discrete-time system model and observation model can be written as:

$$
\begin{aligned}
\boldsymbol{x}_{\boldsymbol{k}+\mathbf{1}} & =\boldsymbol{\Phi}_{\boldsymbol{k}} \boldsymbol{x}_{\boldsymbol{k}}+\boldsymbol{\Gamma}_{\boldsymbol{k}} \boldsymbol{u}_{\boldsymbol{k}}+\boldsymbol{w}_{\boldsymbol{k}} \\
\boldsymbol{y}_{\boldsymbol{k}} & =h\left(\boldsymbol{x}_{\boldsymbol{k}}\right)+\boldsymbol{v}_{\boldsymbol{k}}
\end{aligned}
$$

$$
\begin{aligned}
& \boldsymbol{\Phi}_{\boldsymbol{k}}=\left[\begin{array}{ccc}
1 & t_{k+1}-t_{k} & 0 \\
0 & 1 & 0 \\
0 & 0 & 1
\end{array}\right], \boldsymbol{\Gamma}_{\boldsymbol{k}}=\left[\begin{array}{c}
\left(t_{k+1}-t_{k}\right)^{2} / 2 \\
t_{k+1}-t_{k} \\
0
\end{array}\right] \\
& h\left(\boldsymbol{x}_{\boldsymbol{k}}\right)=\frac{V_{M k}}{X_{F k}-X_{M k}}
\end{aligned}
$$

$\boldsymbol{x}_{\boldsymbol{k}}$ is the state vector, $\boldsymbol{x}_{\boldsymbol{k}}=\left[X_{M k}, V_{M_{k}}, X_{F_{k}}\right]^{T}, \boldsymbol{y}_{\boldsymbol{k}}$ is the observation vector, $\boldsymbol{y}_{\boldsymbol{k}}=O F D_{k}$. $\boldsymbol{w}_{\boldsymbol{k}}$ and $\boldsymbol{v}_{\boldsymbol{k}}$ are the process and observation noise vectors respectively. We assume they are zero mean Gaussian processes with covariance matrices $\boldsymbol{Q}$ and $\boldsymbol{R}$ which are experimentally tuned for optimal EKF performance.

The EKF follows the steps below:

$$
\begin{aligned}
& \hat{\boldsymbol{x}}_{\boldsymbol{k}+\mathbf{1} \mid \boldsymbol{k}}=\boldsymbol{\Phi}_{\boldsymbol{k}} \hat{\boldsymbol{x}}_{\boldsymbol{k} \mid \boldsymbol{k}}+\boldsymbol{\Gamma}_{\boldsymbol{k}} a \\
& \boldsymbol{P}_{\boldsymbol{k}+\mathbf{1} \mid \boldsymbol{k}}=\boldsymbol{\Phi}_{\boldsymbol{k}} \boldsymbol{P}_{\boldsymbol{k} \mid \boldsymbol{k}} \boldsymbol{\Phi}_{\boldsymbol{k}}^{T}+\boldsymbol{Q} \\
& \boldsymbol{H}_{\boldsymbol{k}+\mathbf{1}}=\left.\frac{\partial h}{\partial \boldsymbol{x}}\right|_{\hat{\boldsymbol{x}}_{\boldsymbol{k}+\mathbf{1} \mid \boldsymbol{k}}}=\left[\begin{array}{c}
\frac{\hat{V}_{M_{k+1 \mid k}}}{\left(\hat{X}_{F_{k+1 \mid k}}-\hat{X}_{M_{k+1 \mid k}}\right)^{2}} \\
\frac{1}{\hat{X}_{F_{k+1 \mid k}}-\hat{X}_{M_{k+1 \mid k}}} \\
\frac{-\hat{V}_{M_{k+1}}}{\left(\hat{X}_{F_{k+1 \mid k}}-\hat{X}_{\left.M_{k+1 \mid k}\right)^{2}}\right.}
\end{array}\right]^{T} \\
& \boldsymbol{K}_{\boldsymbol{k}+\mathbf{1}}=\frac{\boldsymbol{P}_{\boldsymbol{k}+\mathbf{1} \mid \boldsymbol{k}} \boldsymbol{H}_{\boldsymbol{k}+\mathbf{1}}^{T}}{\boldsymbol{H}_{\boldsymbol{k}+\mathbf{1}} \boldsymbol{P}_{\boldsymbol{k}+\mathbf{1} \mid \boldsymbol{k}} \boldsymbol{H}_{\boldsymbol{k}+\mathbf{1}}^{T}+R} \\
& \hat{\boldsymbol{x}}_{\boldsymbol{k}+\mathbf{1} \mid \boldsymbol{k}+\mathbf{1}}=\hat{\boldsymbol{x}}_{\boldsymbol{k}+\mathbf{1} \mid \boldsymbol{k}}+\boldsymbol{K}_{\boldsymbol{k}+\mathbf{1}}\left(O F D_{k+1}-h\left(\hat{\boldsymbol{x}}_{\boldsymbol{k}+\mathbf{1} \mid \boldsymbol{k}}\right)\right) \\
& \boldsymbol{P}_{\boldsymbol{k}+\mathbf{1} \mid \boldsymbol{k}+\mathbf{1}}=\left(\boldsymbol{I}-\boldsymbol{K}_{\boldsymbol{k}+\mathbf{1}} \boldsymbol{H}_{\boldsymbol{k}+\mathbf{1}}\right) \boldsymbol{P}_{\boldsymbol{k}+\mathbf{1} \mid \boldsymbol{k}}
\end{aligned}
$$

where $\boldsymbol{P}$ is the state error covariance matrix, $\boldsymbol{H}$ is the Jacobian matrix to linearize the observation model, and $\boldsymbol{K}$ is the Kalman gain.

\section{B. Nonlinear Observability of the EKF}

As the observation model is nonlinear, we use the Lie derivatives of 16 with respect to the system model dynamics in 15 to construct the observability matrix $\mathcal{O}$.

$$
\begin{aligned}
& \mathcal{O}=\left[\begin{array}{lll}
\mathcal{L}_{\dot{x}}^{0} h(\boldsymbol{x}) & \mathcal{L}_{\dot{x}}^{1} h(\boldsymbol{x}) & \mathcal{L}_{\dot{x}}^{2} h(\boldsymbol{x})
\end{array}\right]^{T} \\
& =\left[\begin{array}{c}
V_{M} /\left(X_{F}-X_{M}\right) \\
V_{M}^{2} /\left(X_{F}-X_{M}\right)^{2}+a /\left(X_{F}-X_{M}\right) \\
2 V_{M}^{3} /\left(X_{F}-X_{M}\right)^{3}+3 V_{M} a /\left(X_{F}-X_{M}\right)^{2}
\end{array}\right]
\end{aligned}
$$

Observability of the system is determined by the rank of the Jacobian of matrix $\mathcal{O}$. As $X_{F}$ and $X_{M}$ always appear in a pair with opposite signs in the denominator in (27), the Jacobian will not be full rank. Thus, $X_{F}$ and $X_{M}$ are not observable. The OFD measurement only provides information on the relative distance but not on the absolute location of both. The model input updates $X_{M}$ from an arbitrary initial state and does not update $X_{F}$. However, our method is still valid as the distance $X_{F}-X_{M}$ and $V_{M}$ are still observable under the condition that $a \neq 0, V_{M} \neq 0$, and $X_{F}-X_{M} \neq 0$.

\section{Numerical Investigations}

The EKF was validated by numerical simulations in MATLAB (Fig. 6). We generate a time series of acceleration, the corresponding velocity, and MAV position $X_{M_{T}}$. Knowing the velocity of the MAV and the geometry of the corridor 

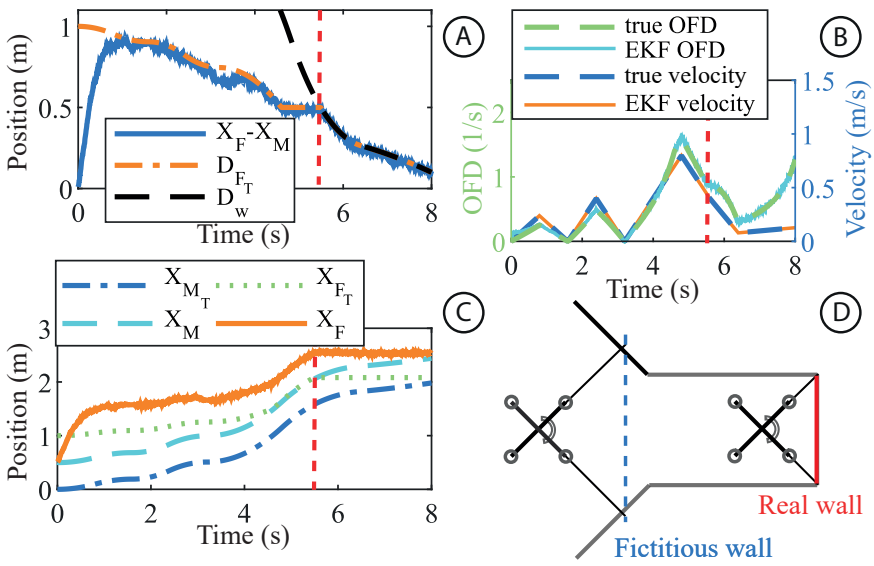

Fig. 6. (A) The comparison between EKF estimated fictitious distance $X_{F}-$ $X_{M}$, the theoretical fictitious distance $D_{F_{T}}$, and the real distance to final obstacle $D_{w}$. (B)(C) The theoretical and estimated MAV and fictitious wall positions, OFD, and velocity. (D) A sketch of the changing width corridor used in the simulation. The red dash line indicates when the real wall is observed by the DOFP. The individual FoVs of the OF sensors are ignored.

(Fig. 6D), the theoretical value of the angular flow rate of both OF sensors can be calculated following (3). The theoretical OFD is calculated with (7), and the theoretical fictitious distance $D_{F_{T}}$ is obtained by (14). The ground truth of the fictitious wall position $X_{F_{T}}=X_{M_{T}}+D_{F_{T}}$. Gaussian noise is added to the given acceleration and the true OFD. Then these two signals are used as the model input and the measurement update of the EKF respectively.

The initial $X_{F}$ and $X_{M}$ were set to be different from the theoretical values. The non-observability of $X_{F}$ and $X_{M}$ is apparent in Fig. 6C; the estimated values deviate from the theoretical values by the initial value of $X_{M}-X_{M_{T}}$. The distance estimation converged to the theoretical value after $1 \mathrm{~s}$ and estimated the distance to wall $D_{w}$ accurately after $5.5 \mathrm{~s}$ (Fig. 6A). The velocity and OFD are also accurately estimated (Fig. 6B). Large OFD values can occur before the MAV sees the wall. This would be a false positive if a simple OFD threshold was used for obstacle detection. Although the fictitious wall position is relative to the initial conditions, the incoming obstacle is still indicated by when the fictitious wall stops moving forward, which is also after $5.5 \mathrm{~s}$.

\section{Obstacle Detection Criterion}

When the fictitious wall moves along with the MAV, there is no obstacle. Statistically, one could use the gradient of the fictitious wall position to detect the obstacle. A near 0 gradient can be the criterion for obstacle observation.

In practice, numerous factors could degrade the accuracy of the EKF and the effectiveness of this criterion, including but not limited to, the IMU noise and bias, the DOFP errors due to the lack of visual features, and unrepresentative noise matrices. We found that the $X_{F}$ curve may not be perfectly flat when a real obstacle is observed (Fig. 7B). We need to detect a near 0 band of the gradient for a continuous period of time to ensure that the obstacle is real. This causes delayed detections.
The product of gradients $(\mathrm{PoG})$ of the MAV position and distance is a more obvious critical condition for detection (Fig. 7B). When the MAV flies forward and the fictitious distance decreases, PoG becomes negative, which indicates an obstacle. We can set a negative PoG threshold value as the detection criterion.

However, the PoG method is not applicable in some circumstances. An example of this is a flight through increasingly narrow corridors (Fig. 6). $D_{F}$ would decrease while the MAV position moves forward. The PoG is negative despite no obstacle in front. Although PoG gave a false signal, the negative value still accurately indicated the increased risk of collision. Other than the narrowing corridor case, we also found that PoG could be negative if we halt the MAV during a forward flight. This is mitigated by reinitializing the EKF when the MAV hovers and adding the condition: $V_{M}>0.1 \mathrm{~m} \mathrm{~s}^{-1}$.

An obstacle becoming observable does not mean that a collision is imminent. A distance threshold is added to trigger the emergency action once the obstacle detected (Fig. 57.

\section{E. Limitations of the Proposed Approach}

The definition of the fictitious wall has limitations in certain scenarios. OFD should be the ratio of the relative velocity to the relative distance. As the fictitious wall is static, we define the observation model of the EKF using the ratio of MAV velocity over $D_{F}$. However, if the real obstacle is moving, the EKF becomes invalid. For example, when the MAV flies closely behind an obstacle moving at the same speed, there is no relative motion between the two. Therefore, the DOFP will generate a near-zero OFD. However, based on (16), the OFD should not be zero when MAV velocity is not zero. The observation model and the measurement are in conflict. This is a model-plant mismatch problem.

If we set the time derivative of the fictitious wall position as velocity $V_{F}$, the observation model in EKF becomes $O F D=$ $\frac{V_{M}-V_{F}}{X_{F}-X_{M}}$. As discussed, the fictitious wall moves along with the MAV when only the surroundings are observed, $V_{M} \approx V_{F}$, which means the OFD by observation model would be around 0 . However, the real OFD updated from DOFP will not be zero. Another model-plant mismatch problem occurred. This explains why the fictitious wall has zero velocity.

\section{RESULTS AND DISCUSSIONS}

\section{A. System Validation Tests}

Flight tests were conducted with a mocap system for position control before the emergency action and ground truth data collection. As these tests were intended to validate the EKF and the obstacle detection criterion, an idealized test environment was constructed; a $2 \mathrm{~m}$ wide textured vertical wall was used as the obstacle. The wall comes within the MAV's FoV at around $1.5 \mathrm{~m}$ away.

The fictitious distance was estimated accurately, and the fictitious wall position stayed relatively constant after the obstacle was detected (Fig. 7A). However, the $D_{F}$ and $X_{F}$ drift when the MAV is static. This is due to the EKF being unobservable when the acceleration, velocity, or OFD are zero. Nonetheless, as we only need the gradient of the EKF output, 

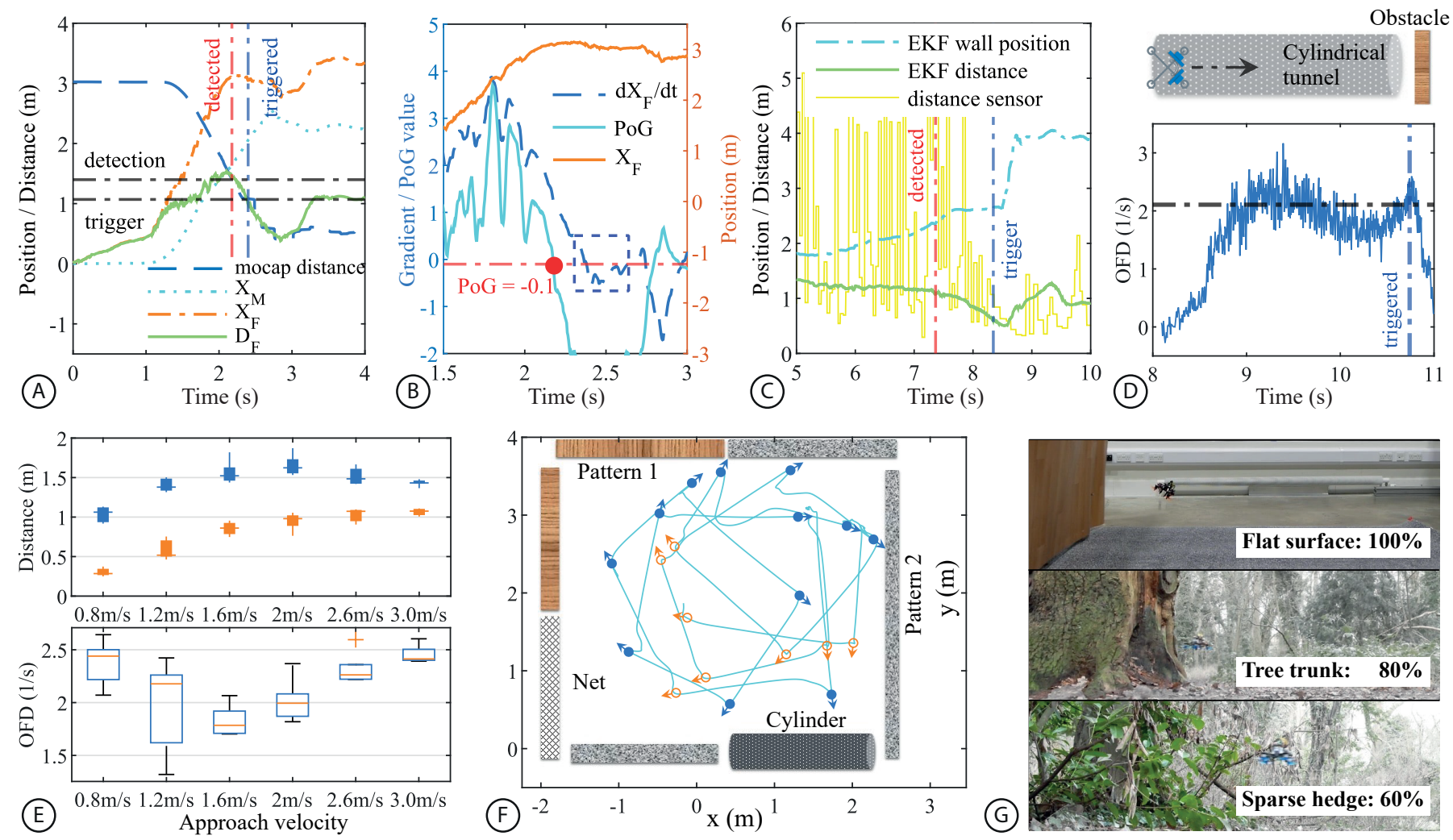

Fig. 7. (A) Graph of a lab environment flight test with the emergency trigger distance at $1 \mathrm{~m}$. The detected time, triggered time, and the distance at detection are noted with dash-dot lines. The wall becomes observable at $1.40 \mathrm{~m}$, and braking was triggered at $1.07 \mathrm{~m}$. After triggering, the MAV stabilized at approximately $0.6 \mathrm{~m}$ away from the wall. (B) The obstacle detection criterion comparison between gradients of fictitious wall position and the PoG from the test shown in (A). The PoG threshold (red dash-dot line), the trigger point (red dot), and the near-zero band of gradient detection window (blue box) are shown. (C) A comparison of the EKF estimated distance and the VL53L1X distance sensor when the MAV flies towards a sparse hedge. (D) DOFP OFD during the flight within a cylindrical tunnel (setup shown above the graph). Trigger time and OFD are noted with dash-dot lines. (E) Box plots of detection distances (blue filled boxes), trigger distances (orange filled boxes), and OFDs from mocap at trigger on different approach velocities. Five or more tests were completed for each velocity. (F) The flight path on the horizontal plane, captured by mocap, and the MAV direction at trigger during the initial $60 \mathrm{~s}$ of the autonomous obstacle avoidance test. The true and false positive triggers are indicated by blue and orange markers respectively. (G) The photos and the collision prevention successful rate when MAV flew into indoor flat surface, tree trunk, and sparse hedge. Ten tests or more were conducted for each scenario.

and the near-obstacle distance is estimated accurately, the EKF is competent for obstacle detection and emergency triggering.

The system is also validated against different approaching velocities (Fig. 7 7E). The results showed consistent detection and trigger distances at velocities ranging from $0.8 \mathrm{~m} \mathrm{~s}^{-1}$ to $3 \mathrm{~m} \mathrm{~s}^{-1}$. The detection and the trigger distances were smaller when flying at $0.8 \mathrm{~m} \mathrm{~s}^{-1}$ and $1.2 \mathrm{~m} \mathrm{~s}^{-1}$, and were around $1.5 \mathrm{~m}$ and $1 \mathrm{~m}$ respectively when the velocity was larger than $1.6 \mathrm{~m} \mathrm{~s}^{-1}$. The EKF is more accurate with a greater observed motion by the OF sensor; low flight speed increases estimation errors. Nevertheless, the detection, triggering, and deceleration were successful in all thirty flight tests without re-tuning which shows the adaptability and resilience of the proposed method. At the trigger distance of $1 \mathrm{~m}$, the maximum successful approaching velocity achieved is around $3 \mathrm{~m} \mathrm{~s}^{-1}$.

The ground truth OFD when the emergency action was triggered was not consistent and it varied with the velocity (Fig. 7E). This affirms that the single OFD threshold obstacle detection criterion can only be used for constant velocity flight. We also conducted flights inside a blocked cylindrical tunnel (Fig. 7D), similar to the simulation settings in Section V-C The trigger OFD is smaller than the maximum OFD before the triggering. These tests validate that our method can prevent false alarms as compared to the single OFD threshold method.

\section{B. Robustness in Off-design Conditions}

In order to test the robustness of the system, flights of incidence angles $\gamma$ from $0^{\circ}$ to $90^{\circ}$ in $15^{\circ}$ intervals were conducted. The obstacle is a $3.5 \mathrm{~m}$ wide, $1.2 \mathrm{~m}$ high vertical wall and the approach speed is approximately $2.5 \mathrm{~m} \mathrm{~s}^{-1}$.

Ten tests were conducted at each $\gamma$. At $\gamma \geq 66^{\circ}$, one of the OF sensor will not see the wall. This should invalidate the OFD estimation by DOFP. However, the system achieved $100 \%$ successful rate at $75^{\circ}$, and the maximum achievable angle is $80^{\circ}$, at which the MAV flew almost parallel to the obstacle. At $92.5^{\circ}$, the MAV flew nearby the obstacle without triggering emergency action.

As explained in Section III-C, the estimated and the real OFDs differ by a constant ratio. Although the EKF estimation is inaccurate, the PoG can still become negative when the MAV is near the obstacle. The estimated distances were smaller than $1 \mathrm{~m}$ when the obstacle was detected, and the collision prevention was triggered immediately post-detection. 
Multiple obstacle avoidance events on a single mission were tested by an onboard forward-trigger-turn loop. The MAV flew continuously inside a room with two different-patterned walls, a cylindrical structure, and nets (Fig.7F); the command velocity was $1.5 \mathrm{~m} \mathrm{~s}^{-1}$; the test lasted approximately $150 \mathrm{~s}$. At times the MAV triggered earlier than expected, these are labeled as false positive triggers. In total, there were 42 triggers, 13 of which were false positives, achieving a $69 \%$ true positive rate. Based on where the false positives occurred, the possible reasons include the increasingly narrow corridor (corners) cases mentioned in Section V-C, and the inaccurate OFD estimation when DOFP facing the cylinder structure and nets. The collision occurred when MAV was too close to the surface after turning and the EKF did not have sufficient time to converge.

\section{Natural Environment Tests and Statistical Evaluations}

Field tests in challenging environments showed varying degrees of success (Fig. $7 \mathrm{G}$ ). To test the practicability of the system, the MAV was manually piloted in windy conditions, and flown toward sparse woodland hedgerows and tree trunks. The prevention success rate varied with the sparseness and the texture of the obstacle. At times, the MAV was able to fly through the hedgerows without collisions or triggering avoidance actions. Thus, the system is potentially capable of operating in cluttered environment.

For comparison, a front facing distance sensor in the same weight range as the OF sensors was installed on the MAV when testing against sparse hedgerows. The distance sensor was deemed too noisy and inaccurate (Fig. 7F).

\section{CONCLUSION}

An onboard lightweight obstacle detection solution for MAVs was presented. The OFD, combined with inertial measurements in an EKF, enabled a $158 \mathrm{~g}$ quadrotor to detect and maintain a safe distance from frontal obstacles. Improving upon the use of OFD, a relative value, as a stopping metric, the EKF allows the estimation of the distance to the obstacle. Using our fictitious wall model, we can differentiate between far-field and imminent obstacles. This detection method allows MAV to fly safely in a broader velocity range. Simulations, idealized indoor tests, and field tests were conducted to benchmark the capabilities of the $20 \mathrm{~g}$ sensor suite. The system's mass makes it attractive to be combined with tactile sensors to form holistic solutions for sensing, impact protection, and surface interaction for MAVs [22], [23].

For future work, a $360^{\circ} \mathrm{OF}$ sensor suite will increase the fidelity and robustness of detection in all planar directions (Fig. 1D). Two OF sensors facing the flight direction will estimate the obstacle position, while the others, measuring larger OF, would help to provide better OFD estimations. In addition, the model-plant mismatch will be studied to mitigate the false positive rate.

\section{ACKNOWLEDGMENT}

The authors would like to thank Dr. Sophie Armanini, Dr. Salua Hamaza and Mr. Hussain Noor for proof-reading this paper, and Miss Nan Zhang for assisting on the fieldwork. We thank Prof. Holger Krapp and Dr. Franck Ruffier for their guidance and inspiration.

\section{REFERENCES}

[1] Y. K. Kwag and C. H. Chung, "UAV based collision avoidance radar sensor,' in International Geoscience and Remote Sensing Symposium (IGARSS), 2007, pp. 639-642.

[2] R. Sabatini, "Development and Flight Test of an Avionics Lidar for Helicopter and UAV Low-Level Flight," Journal of Aeronautics \& Aerospace Engineering, vol. 02, no. 03, 2013.

[3] W. G. Aguilar, V. P. Casaliglla, and J. L. Polit, "Obstacle Avoidance for Low-Cost UAVs," in Proc. IEEE International Conference on Semantic Computing (ICSC'17), mar 2017, pp. 503-508.

[4] H. D. Escobar-Alvarez, N. Johnson, T. Hebble, K. Klingebiel, S. A Quintero, J. Regenstein, and N. A. Browning, "R-advance: Rapid adaptive prediction for vision-based autonomous navigation, control, and evasion," Journal of Field Robotics, vol. 35, no. 1, pp. 91-100, 2018.

[5] D. Falanga, S. Kim, and D. Scaramuzza, "How Fast Is Too Fast? the Role of Perception Latency in High-Speed Sense and Avoid," IEEE Robotics and Automation Letters, vol. 4, no. 2, pp. 1884-1891, 2019.

[6] M. V. Srinivasan, S. Zhang, and J. S. Chahl, "Landing strategies in honeybees, and possible applications to autonomous airborne vehicles," The Biological Bulletin, vol. 200, no. 2, pp. 216-221, 2001.

[7] J. J. Koenderink and A. J. van Doorn, "Facts on optic flow," Biological cybernetics, vol. 56, no. 4, pp. 247-254, 1987.

[8] J. Lecoeur, M. Dacke, D. Floreano, and E. Baird, "The role of optic flow pooling in insect flight control in cluttered environments," Scientific Reports, vol. 9, no. 1, p. 7707, 2019.

[9] K. McGuire, G. De Croon, C. De Wagter, K. Tuyls, and H. Kappen, "Efficient Optical Flow and Stereo Vision for Velocity Estimation and Obstacle Avoidance on an Autonomous Pocket Drone," IEEE Robotics and Automation Letters, vol. 2, no. 2, pp. 1070-1076, apr 2017.

[10] R. C. Nelson and J. Aloimonos, "Obstacle avoidance using flow field divergence," IEEE Transactions on pattern analysis and machine intelligence, vol. 11, no. 10, pp. 1102-1106, 1989.

[11] J. R. Serres and F. Ruffier, "Biomimetic Autopilot Based on Minimalistic Motion Vision for Navigating along Corridors Comprising U-shaped and S-shaped Turns," Journal of Bionic Engineering, 2015.

[12] G. de Croon, C. De Wagter, and T. Seidl, "Enhancing optical-flow-based control by learning visual appearance cues for flying robots," Nature Machine Intelligence, vol. 3, no. 1, pp. 33-41, 2021.

[13] G. Cho, J. Kim, and H. Oh, "Vision-based obstacle avoidance strategies for mavs using optical flows in 3-d textured environments," Sensors, vol. 19, no. 11, p. 2523, 2019.

[14] H. W. Ho, G. C. de Croon, and Q. Chu, "Distance and velocity estimation using optical flow from a monocular camera," International Journal of Micro Air Vehicles, vol. 9, no. 3, pp. 198-208, 2017.

[15] H. W. Ho, G. C. De Croon, E. Van Kampen, Q. P. Chu, and M. Mulder, "Adaptive Gain Control Strategy for Constant Optical Flow Divergence Landing," IEEE Transactions on Robotics, vol. 34, no. 2, pp. 508-516, 2018.

[16] B. Herisse, F.-X. Russotto, T. Hamel, and R. Mahony, "Hovering flight and vertical landing control of a VTOL Unmanned Aerial Vehicle using Optical Flow," in 2008 IEEE/RSJ International Conference on Intelligent Robots and Systems. IEEE, 2008, pp. 801-806.

[17] N. Ancona and T. Poggio, "Optical flow from 1D correlation: Application to a simple time-to-crash detector," in IEEE International Conference on Computer Vision, 1993, pp. 209-214.

[18] T. Poggio, A. Verri, and V. Torre, "Green Theorems and Qualitative Properties of the Optical Flow," MIT, Artificial Intelligent Laboratory, Tech. Rep., apr 1991.

[19] W. E. Green and P. Y. Oh, "Optic-Flow-Based Collision Avoidance," IEEE Robotics Automation Magazine, vol. 15, no. 1, pp. 96-103, 2008.

[20] J. C. Zufferey and D. Floreano, "Fly-Inspired Visual Steering of an Ultralight Indoor Aircraft," IEEE Transactions on Robotics, vol. 22, no. 1, pp. 137-146, 2006.

[21] D. N. Lee, "A Theory of Visual Control of Braking Based on Information about Time-to-Collision," Perception, vol. 5, no. 4, pp. 437-459, 1976.

[22] P. Sareh, P. Chermprayong, M. Emmanuelli, H. Nadeem, and M. Kovac, "Rotorigami: A rotary origami protective system for robotic rotorcraft," Science Robotics, vol. 3, no. 22, 2018.

[23] M. Kovac, "Learning from nature how to land aerial robots," Science, vol. 352, no. 6288, pp. 895-896, 2016. 